\title{
Inulin prebiotic: is it all about bifidobacteria?
}

Article

Accepted Version

Claus, S. (2017) Inulin prebiotic: is it all about bifidobacteria? Gut, 66 (11). pp. 1883-1884. ISSN 1468-3288 doi: https://doi.org/10.1136/gutjnl-2017-313800 Available at https://centaur.reading.ac.uk/70004/

It is advisable to refer to the publisher's version if you intend to cite from the work. See Guidance on citing.

To link to this article DOI: http://dx.doi.org/10.1136/gutjnl-2017-313800

Publisher: BMJ Publishing

All outputs in CentAUR are protected by Intellectual Property Rights law, including copyright law. Copyright and IPR is retained by the creators or other copyright holders. Terms and conditions for use of this material are defined in the End User Agreement.

\section{www.reading.ac.uk/centaur}

\section{CentAUR}

Central Archive at the University of Reading

Reading's research outputs online 
Inulin prebiotic: is it all about bifidobacteria?

Sandrine Paule Claus*

* Dept of Food and Nutritional Sciences, Whiteknights campus, The University of Reading, PO Box 226, Reading RG6 6AP, UK.

s.p.claus@reading.ac.uk

Tel: $+44(0) 1183788717$

Key words: Prebiotic, colonic microflora, bifidobacteria

Word count: 1122 
In this issue, Vandeputte et al. present a unique study exploring the effects of chicory-derived inulin on the human gut microbiota by combining Next Generation Sequencing and fecal metabolomics to gain new insights into the mechanisms of action underlying inulin health promoting effects.[1] The study supports that inulin prebiotics stimulate growth of bifidobacteria while simultaneously driving a number of other modulations of the gut microbial ecosystem that may be associated with health-promoting effects.

Consumption of inulin-type fructans (ITFs) has been associated with a number of health benefits, including normalisation of gastrointestinal function, regulation of body weight gain and energy metabolism. [2] Some of the suggested mechanisms of action include regulation of the immune system, modulation of gastrointestinal peptides, production of SCFAs and modulation of triglyceride metabolism. [2-4] Down in the intestine, ITFs have long been known for their prebiotic activity targeted towards the enhancement of the genus bifidobacteria.[5] The later is a well-known inhabitant of the human gut and one of the first micro-organism to colonize the infant's intestine at birth. Bifidobacteria are believed to promote health by producing some antimicrobial substances protecting the host from opportunistic pathogens, in addition to large amounts of lactic acid, which contribute to maintain a low $\mathrm{pH}$ in the colon thereby limiting pathogen growth. Yet, inulin prebiotic effects on human's gut microbial ecology have so far mostly been assessed using culture-based methods or targeted quantitative PCR (Polymerase Chain Reaction) and FISH (Fluorescent In Situ Hybridization), which limited our ability to assess the full extent of their impact on the gut microbial community. In this issue, Vandeputte et al. filled 
this caveat by applying for the first time a Next Generation Sequencing technology to capture the untargeted impact of inulin prebiotic consumption on the human gut microbiota.[1]

In this double blind, randomized, placebo-controlled, cross-over diet intervention study, 44 healthy volunteers with constipation consumed either a chicory-derived inulin mix or maltodextrin for 4 weeks on a daily basis. The full details of the original experimental design and results on gastrointestinal parameters were published previously,[6] and indicated that inulin intake significantly improved stool frequency. The present study reports the results of the gut microbiota phylogenetic analysis complemented by some fecal metabolomics data. Focusing on the V4 hypervariable region of the $16 \mathrm{~S}$ rDNA, the authors first demonstrated that inulin intake shifted the gut microbial composition towards a significant increase of Bifidobacterium and Anaerostipes spp. concomitant with a reduction in Bilophila population. Although an increase in bifidobacteria has been consistently observed in association with ITFs consumption, this is the second report of an increase in Anaerostipes spp. in humans.[3] Indeed, Dewulf et al. reported a number of inulin-induced microbial modulations in obese women, including an increase in Anaerostipes caccae, Lactobacillus gasseri and Faecalibacterium prausnitzii populations.[3] These are important findings because some Anaerostipes species and Faecalibacterium prausnitzii have been reported to be butyrate producers and therefore of potential health benefit for the host. $[7,8]$

A reduction in Bilophila population is also an interesting outcome because these bacteria of the Desulfivibrionacaeae family are sulfite reducing organisms known to 
produce hydrogen sulphide $\left(\mathrm{H}_{2} \mathrm{~S}\right)$ toxic for the host.[9] In addition, the change in Bilophila population was significantly correlated with discomfort; the bigger its reduction, the lower discomfort score was reported. This indicates that some of the potentially beneficial effects of ITFs may be mediated by the selective reduction of Bilophila spp. in the gut microbial community. Interestingly, a similar decrease in Bilophila spp. following inulin intake had been reported in rodents using a similar deep sequencing approach, where it was also associated with improved gut barrier function.[10] Previous studies in human volunteers have reported various inulin- and FOS-triggered modulations of gut bacterial groups independent of bifidobacteria (reviewed by Gibson et al.[11]), of which a decrease in enterobacteria populations that also contain a number of opportunistic pathogens.[11] Altogether, this suggests that inulin may provide health benefits by promoting a colonic environment unfavourable for some pathogens.

In this context, it is pertinent to get an insight into the modulations of the gut luminal environment, which may be modified as a result of an alteration of gut microbial metabolic activity. The authors therefore investigated the fecal metabolome following inulin intake by GC-MS (Gas Chromatography coupled with Mass Spectrometry). Unlike other studies that captured an increase in short chain fatty acids following inulin intake, no effects on known metabolic products of bifidobacteria were reported here. Nevertheless, the particular GC-MS protocol used for the metabolomics analysis in this study did not allow detection of a number of major bacterial metabolites, such as lactic acid. This constitutes the main limitation of the study and hence it precludes any conclusion about the influence of the 
prebiotic treatment over gut bacterial metabolism. This is regrettable since a proper evaluation of functional modulations of active ingredients designed to target the gut microbial ecosystem is necessary to assess their potential as a modulator of microbiota function. Indeed, although it is possible to infer the metabolic potential of a microbial ecosystem based on its metagenome, only a direct measurement of the metabolites released by the microbial population can accurately reflect the metabolic adjustments of the micro-organisms to a diet challenge. Such information is useful to control for inter-individual variability to diet interventions, as we must be able to measure an individual's response to functional foods to ultimately determine the individual health benefit. Exploring metabolic variations in a wider range of biological compartments, such as urine where many microbial co-metabolites accumulate, would also be helpful to depict a more accurate picture of the hostmicrobiota metabolic response to a prebiotic intervention.

So is inulin prebiotic all about bifidobacteria? On one hand, this study confirms that the invariable bacterial response to inulin challenge remains an increase in bifidobacteria populations, which has been consistently reported over the last 20 years. On the other hand, it suggests that these effects are accompanied by other ecological changes that should not be disregarded for their health-promoting consequences, which argues in favour of a broader definition of prebiotics as previously suggested.[12] It is likely that the reason why we have not been able to consistently report such alterations is due to the fact that this aspect of the microbial response to inulin is more prone to inter-individual variations and could not be 
captured using targeted microbiological methods. Yet, it appears that these

idiosyncratic bacterial community shifts deserve serious consideration.

In conclusion, the work of Vandeputte et al.[1] brings us one step closer to designing personalised diets based on an individual predictable response to prebiotics. Future studies will largely benefit from using a similar holistic approach based on a comprehensive assessment of an individual's metabolism combining multiple 'omics' technologies that provide insights into an individual's genome, microbiome and metabolome.

\section{References}

1 Vandeputte D, Falony G, Vieira-Silva S, et al. Prebiotic inulin-type fructans induce specific changes in the human gut microbiota. Gut 2017;:gutjnl-2016313271. doi:10.1136/gutjnl-2016-313271

2 Meyer D, Stasse-Wolthuis M. The bifidogenic effect of inulin and oligofructose and its consequences for gut health. Eur J Clin Nutr 2009;63:1277-89. doi:10.1038/ejcn.2009.64

3 Dewulf EM, Cani PD, Claus SP, et al. Insight into the prebiotic concept: lessons from an exploratory, double blind intervention study with inulin-type fructans in obese women. Gut 2013;62:1112-21. doi:10.1136/gutjnl-2012-303304

$4 \quad$ Cani PD, Dewever C, Delzenne NM. Inulin-type fructans modulate gastrointestinal peptides involved in appetite regulation (glucagon-like peptide1 and ghrelin) in rats. Br J Nutr 2004;92:521-6.

5 Gibson GR, Beatty ER, Wang X, et al. Selective stimulation of bifidobacteria in the human colon by oligofructose and inulin. Gastroenterology 1995.

6 Micka A, Siepelmeyer A, Holz A, et al. Effect of consumption of chicory inulin on bowel function in healthy subjects with constipation: a randomized, doubleblind, placebo-controlled trial. Int J Food Sci Nutr 2016;68:82-9. doi:10.1080/09637486.2016.1212819

7 Schwiertz A, Hold GL, Duncan SH, et al. Anaerostipes caccae gen. nov., sp. nov., a New Saccharolytic, Acetate-utilising, Butyrate-producing Bacterium from Human Faeces. Systematic and Applied Microbiology 2002;25:46-51. 
doi:10.1078/0723-2020-00096

8 Allen-Vercoe E, Daigneault M, White A, et al. Anaerostipes hadrus comb. nov., a dominant species within the human colonic microbiota; reclassification of Eubacterium hadrum Moore et al. 1976. Anaerobe 2012;18:523-9. doi:10.1016/j.anaerobe.2012.09.002

9 Carbonero F, Benefiel AC, Alizadeh-Ghamsari AH, et al. Microbial pathways in colonic sulfur metabolism and links with health and disease. Front Physiol 2012;3:448. doi:10.3389/fphys.2012.00448

10 Everard A, Lazarevic V, a NGI, et al. Microbiome of prebiotic-treated mice reveals novel targets involved in host response during obesity. The ISME Journal Published Online First: 3 April 2014. doi:10.1038/ismej.2014.45

11 Gibson GR, Scott KP, Rastall RA, et al. Dietary prebiotics: current status and new definition. Food Science \& Technology Bulletin: Functional Foods 2010;7:1-19. doi:10.1616/1476-2137.15880

12 Bindels LB, Delzenne NM, Cani PD, et al. Towards a more comprehensive concept for prebiotics. Nat Rev Gastroenterol Hepatol 2015;12:303-10. doi:10.1038/nrgastro.2015.47 